Published in final edited form as:

Nat Biomed Eng. 2016 ; 1: . doi:10.1038/s41551-016-0004.

\title{
Solid stress and elastic energy as measures of tumour mechanopathology
}

\author{
Hadi T. Nia ${ }^{1}$, Hao Liu ${ }^{1,2}$, Giorgio Seano ${ }^{1}$, Meenal Datta ${ }^{1,3}$, Dennis Jones ${ }^{1}$, Nuh Rahbari ${ }^{1}$, \\ Joao Incio ${ }^{1,4}$, Vikash P. Chauhan ${ }^{1}$, Keehoon Jung ${ }^{1}$, John D. Martin ${ }^{1}$, Vasileios \\ Askoxylakis ${ }^{1}$, Timothy P. Padera ${ }^{1}$, Dai Fukumura1 ${ }^{1}$, Yves Boucher ${ }^{1}$, Francis J. Hornicek ${ }^{5}$, \\ Alan J. Grodzinsky6 ${ }^{6}$, James W. Baish ${ }^{7}$, Lance L. Munn ${ }^{1}$, and Rakesh K. Jain ${ }^{1}$ \\ ${ }^{1}$ Edwin L. Steele Laboratories, Department of Radiation Oncology, Massachusetts General \\ Hospital, Harvard Medical School, Boston, MA 02114, USA \\ ${ }^{2}$ Leder Human Biology and Translational Medicine, Biology and Biomedical Sciences, Harvard \\ Medical School, Boston MA 02115, USA \\ ${ }^{3}$ Department of Chemical and Biological Engineering, Tufts University, Medford, MA 02155, USA \\ ${ }^{4}$ Department of Internal Medicine, Hospital S. Joao; I3S, Institute for Innovation and Research in \\ Health; and Faculty of Medicine, Porto University; Porto, Portugal \\ ${ }^{5}$ Orthopedic Oncology Service, Center for Sarcoma and Connective Tissue Oncology, \\ Massachusetts General Hospital, Harvard Medical School, Boston, MA 02114, USA \\ ${ }^{6}$ Center for Biomedical Engineering, Departments of Mechanical, Electrical and Biological \\ Engineering, Massachusetts Institute of Technology, Cambridge, MA 02139, USA \\ ${ }^{7}$ Department of Biomedical Engineering, Bucknell University, Lewisburg, PA 17837, USA
}

\begin{abstract}
Solid stress and tissue stiffness affect tumour growth, invasion, metastasis and treatment. Unlike stiffness, which can be precisely mapped in tumours, the measurement of solid stresses is challenging. Here, we show that two-dimensional spatial mappings of solid stress and the resulting elastic energy in excised or in situ tumours with arbitrary shapes and wide size ranges can be obtained via three distinct and quantitative techniques that rely on the measurement of tissue displacement after disruption of the confining structures. Application of these methods in models of primary tumours and metastasis revealed that: (i) solid stress depends on both cancer cells and their microenvironment; (ii) solid stress increases with tumour size; and (iii) mechanical confinement by the surrounding tissue significantly contributes to intratumoural solid stress. Further study of the genesis and consequences of solid stress, facilitated by the engineering principles presented here, may lead to significant discoveries and new therapies.
\end{abstract}

Users may view, print, copy, and download text and data-mine the content in such documents, for the purposes of academic research, subject always to the full Conditions of use: http://www.nature.com/authors/editorial_policies/license.html\#termsReprints and permissions information is available online at www.nature.com/reprints

Correspondence to: Rakesh K. Jain. 
Increased tissue stiffness is a widely accepted and actively studied biomechanical property of fibrotic tumours, and has been linked to several hallmarks of cancer, including growth, metabolism, invasion and metastasis [1-7]. The abnormal mechanics of tumours, however, are not limited to tissue stiffening. We recently demonstrated that solid stress represents a new mechanopathology that is consistently elevated in mouse and human tumours [8,9]. This solid stress is distinct from interstitial fluid pressure, as the former is contained in - and transmitted by - solid and elastic elements of the extracellular matrix and cells, rather than fluids [10]. Therefore, tumours are not only more rigid than many normal tissues, but cancer cells also produce and are exposed to these physical forces. Composed of a combination of tension and compression, these forces are significant in tumours, but negligible in most normal tissues.

Early evidence for the existence of solid stress in tumours came from our discovery that blood and lymphatic vessels are mechanically compressed [11-13]; this can contribute to hypoxia $[9,14]$ and promote tumour progression, and lower the efficacy of chemo-, radio-, and immunotherapies [15]. In addition to these physiological consequences, forces can have direct effects on tumour biology; exogenous application of solid stress in vivo can mechanically stimulate tumourigenic pathways, increasing $\beta$-catenin signaling in colon epithelia [16], and controlled application of compressive forces in vitro affects their growth [8] and promotes the collective migration of cancer cells [17]. Strategies designed to reduce solid stress and decompress blood vessels by reducing the levels of collagen and hyaluronic acid (HA) [14, 18, 19], have led to therapeutic approaches for enhancing the efficiency of conventional anti-cancer treatments, and are currently being tested in clinical trials [20,21].

Despite these important implications of solid stress and the immense potential for finding new mechanically-activated pathways and targets, there are currently no high-resolution methods for quantifying solid stress in experimental or human tumours. Unlike stiffness, which can be measured using widely-available, multiscale techniques, measuring solid stress in biological tissues has proven very challenging. Previous studies in our laboratory [8,9], based on Fung's observations that arterial wall tissue relaxes if the contained forces are surgically released [22, 23], demonstrated for the first time, the presence of residual tissue stresses in tumours. However, this approach is based on partial cut through a spherical model of the tumour, which makes the precise release of solid stress and measurement of the deformation challenging. This method is also limited to bulk estimation of solid stress and is not applicable in situ. Alternative imaging-based methods, such as fluorescent oil microdroplet injection [24] and single molecule fluorescent force sensors [25] have optical depth limitations, which restricts their use to cellular and subcellular scale force detection.

Here, we developed the experimental and mathematical frameworks to provide (i) twodimensional mapping of solid stress in tumours (planar cut method), (ii) sensitive estimation of solid stress in small tumours with small magnitudes of solid stress, as is the case for metastatic lesions (slicing method), and (iii) in situ quantification of solid stress in tumours, which retains the effects of the normal surrounding tissues (needle biopsy method). All three methods are based on the concept of releasing the solid stress in a controlled way with defined geometry, and then quantifying the stress-induced deformation by high-resolution ultrasonography or optical microscopy. Given the specific topography of the stress relaxation 
and the geometric and material properties of the tumour, the solid stress and discharged elastic energy is estimated using mathematical modelling. Applying these methods to multiple mouse cancer models in primary and metastatic settings has led to the following findings: (i) solid stress and elastic energy may be different between primary vs. metastatic settings, as they depend on both cancer cells and their microenvironment; (ii) tumours with higher elastic energy are not necessarily stiffer, and the stiffer tumours do not necessarily have higher elastic energy; (iii) solid stress increases with tumour size; and (iv) the normal tissue surrounding a tumour significantly contributes to the intratumoural solid stress.

\section{Two-dimensional (2-D) mapping of solid stress}

To produce a two-dimensional map of solid stress, we made a clean cut through freshly excised tumours, which is embedded in $2 \%$ agarose (Fig. 1A). The planar cut removes the mechanical confinement at the cut surface, and allows this face of the tumour to deform accordingly, as predicted previously by our mathematical model [9]. The deformation is a measure of the stored elastic energy in the tissue caused by the solid stress (Fig. 1B). The deformation $u_{Z}(x, y)$ in the z-direction, imaged over the $x-y$ cross section of the tumour, is an indicator of the magnitude and direction of solid stress in the z-direction at the incision plane: regions with positive deformation (bulged) were under compression, and regions with negative deformation (depressed) were under tension (Fig. 1A, B). For tissues with negligible solid stress, such as normal kidney and liver, deformation was negligible and uniform across the incision surface (Fig. 1C). The deformation is spatially mapped in 3-D by high-resolution ultrasound system (resolution of $30 \mu \mathrm{m}$ ). By developing a mathematical model (3-D finite element model; Fig 1B), the 2-D map of deformation $u_{Z}$ is converted to the strain tensor $\varepsilon_{i j}=1 / 2\left(\partial u_{i} / \partial x_{j}+\partial u_{j} / \partial x_{i}\right)$, from which the stress tensor $\sigma_{\mathrm{ij}}$ is estimated based on Hooke's Law, written in index notation as [26]:

$$
\sigma_{i j}=\frac{E}{(1+\nu)}\left[\varepsilon_{i j}+\frac{\nu}{(1+2 \nu)} \varepsilon_{k k} \delta_{i j}\right]
$$

where $E$ is the modulus of elasticity (stiffness), and $v$ is the Poisson's ratio of the tissue based on linear isotropic assumption, and $\delta_{\mathrm{ij}}$ is the Kroenecker delta. We used the average Young's modulus of the tumour, measured via unconfined compression test in macroscale or via atomic force microscopy measurement at multiple indentation sites. The developed finite element model, however, is capable of incorporating the 3-D stiffness heterogeneities of the tumour previously reported $[27,28]$. Since the cut fully releases the stress component $\sigma_{\mathrm{zz}}$ at the cut plane, we report only this stress component at the cut surface throughout this study. The other stress components such as the stresses in $\mathrm{x}-\mathrm{y}$ plane are also affected and accounted for in the 3-D model. However, since the planar cut method does not fully release these stresses, we do not report them in this study.

Similarly to a spring that is compressed (Fig. 1B), the solid stress field does mechanical work as it accumulates in the tumour, and this work is stored as elastic potential energy during tumour growth. The elastic energy density, $W$, released by the incision is calculated from the following equation for a tumour half with volume $V$ [26]: 


$$
W=\frac{1}{2 V} \int_{V} \sigma_{i j} \varepsilon_{i j} d V
$$

Although the strain energy $W$ is averaged over the volume of the tumour hemisphere, it is not equivalent to the total elastic energy of the tumour. The elastic energy $W$ is only the energy that is released from the relaxation of solid stress at the desired cut plane; for the locations far from the cut plane, the elastic energy and solid stress are not fully released. In order to estimate the total elastic energy in a tumour, the tumour would need to be cut into infinitesimally small pieces so that all of the solid stress and elastic energy is released. The elastic energy is not an independent quantity from the solid stress; however, it describes the tensor-based stress field more sensitively and in the form of a single scalar quantity. Also, elastic energy rigorously differentiates between the forces emanating from solid vs. fluid phases [1] of the tumour based on the following considerations: (i) the interstitial fluid is incompressible, so the fluid pressure cannot do work on the fluid phase of the tumour; and (ii) the solid phase is porous and fluid can flow through it, so the fluid pressure cannot do work on the solid phase of the tumour in quasi-equilibrium events, such as tumour growth.

Using the planar cut method, we mapped the solid stress for three mouse models of orthotopically implanted primary tumours: breast (adenocarcinoma, MMTV-M3C), pancreatic (pancreatic ductal adenocarcinoma (PDAC); AK4.4) and brain (glioblastoma; U87) (Fig. 1D). For each model, a representative ultrasound image shows the stress-induced deformation, quantified with respect to the cut plane (dashed line in yellow). In the lower panel, the 2-D deformations, obtained by quantification of $\delta_{z}$ based on the reference incision plane, from ultrasound images are shown. The 2-D map of solid stress $\sigma_{\mathrm{zz}}$, estimated from the finite element model, and the profile of $\sigma_{\mathrm{zz}}$ across the bisecting dashed lines are shown in the lower panels. The 2-D maps of $\sigma_{\mathrm{zz}}$ in breast and pancreatic tumours show a common pattern of compressive stresses in the central regions, and tensile stresses with higher magnitude, but in narrow peripheral regions. However, in our U87 model of glioblastoma, the tumour experiences compressive stresses over annular regions close to the periphery, rather than the center of the tumour. The maximum value of solid stress varies from $0.21 \mathrm{kPa}$ $(1.56 \mathrm{mmHg})$ in brain tumours to $7 \mathrm{kPa}(52.5 \mathrm{mmHg})$ in pancreatic tumours, which are highly desmoplastic. The planar cut method is capable of estimating a large dynamic range of solid stress, in addition to the variation in the pattern of the compressive and tensile stresses stored in different tumour types. We also demonstrated the cut method on human chondrosarcoma tumours for which the 2-D deformation maps are shown in Fig. S1.

We hypothesized that structural elements, such as collagen fibers, are critical to transmitting solid stress $[9,14]$. To test this hypothesis, we enzymatically depleted collagen in the MMTV-M3C model of breast cancer using collagenase treatment ex vivo. We showed that the average magnitude of solid stress $\left\langle\left|\sigma_{z z}\right|\right\rangle$ was reduced by a factor of more than two, while the elastic energy density $W$ was reduced by a factor of more than six (Fig. 1E). 


\section{Role of microenvironment in the evolution of solid stress}

Little is known about how the biomechanical abnormalities vary between primary and metastatic lesions, or how these differences affect tumour biology. To address this gap, we used the planar cut method to estimate and compare solid stress, elastic energy, and mechanical stiffness in size-matched tumours formed from the same cell lines in primary vs. metastatic sites.

We found that primary PDAC AK4.4 tumours had much larger solid stress levels compared to PDAC liver metastases (Fig. 2A, B), even though both tumours were formed by the same cell line and were approximately size matched. Since these two tumours have similar elastic moduli (Fig. 2E), the higher deformation after incision results in higher stress values (Fig. 2F) and higher elastic energy (Fig. 2G) in primary than liver metastasis. This comparison shows that AK4.4 tumours growing in the pancreas generate higher solid stress and store more elastic energy than AK4.4 liver metastases. Interestingly, for the case of colorectal carcinoma, this trend is reversed (Fig. 2C, D): the primary colorectal tumours have lower levels of solid stress and elastic energy compared to colorectal cancer liver metastases, despite having similar elastic moduli. Our data show that the growth in the liver microenvironment does not always result in lower stored elastic energy. An interesting comparison can also be made between metastases of different cancer cells in the same host organ (in this case, liver). Even though the elastic modulus of PDAC liver metastases is fivefold larger than the colorectal cancer liver metastases, the elastic energy actually shows the opposite trend. This is due to significantly larger stress-induced deformation in colorectal cancer liver metastasis and the higher dependence of elastic energy on stress-induced deformation (Eq. 2). The solid stress shows a lower, but similar trend as the elastic modulus between the two metastases, since solid stress follows a linear relationship with the deformation (Eq. 1). From the above comparisons, we observe that: (i) solid stress and elastic energy are mechanical abnormalities that vary from tumour to the next; (ii) the solid stress and elastic energy depend on both the cancer cells and the microenvironment in which they reside, and (iii) tumours with higher elastic energy are not necessarily stiffer, and the stiffer tumours do not necessarily have higher elastic energy.

\section{Area expansion of a slice is a sensitive index of solid stress}

To measure solid stress and elastic energy in smaller tumours (e.g., in micro- and macrometastases), we developed a more sensitive method -also based on releasing elastic energyby slicing the tissue into thin slices (Fig. 3A). Again, the freshly excised tumour is embedded in $2 \%$ agarose, which is then sliced via vibratome with a desired thickness in the range of 100-500 $\mu \mathrm{m}$. The tumour slice, originally flat, undergoes expansion and contraction in area of the slice when the in-plane stresses and elastic energy are released (Fig. S2), as demonstrated in models of breast tumour (MMTV-M3C), PDAC (AK4.4), and lymph node metastasis of a breast cancer model (4T1) (Fig. 3B-D). Due to inhomogeneities of the inplane stresses (shown for partial release of these stress components in Fig. S3), the slice undergoes out-of-plane bending and buckling. This method is more sensitive than the planar cut method since a thin slice is exposed to more degrees of freedom to deform, and reacts even to small values of solid stress due to small thickness of the slice. The deformed slices 
are then imaged in 3D by high-resolution ultrasound (used here for AK4.4 and MMTV-M3C tumours) or optical frequency domain imaging (OFDI; used here for lymph node metastasis) [29] after stress relaxation. The compressive and tensile stresses (as mapped in Fig. 1D) release in different ways: the compressive forces are released by expansion in volume of the slice, which results in lateral bending and buckling, and the tensile forces are released by pulling the periphery of the tumours inwards. So, the complex geometry that is formed from an originally flat disk-shape slice is the result of non-uniformity and coexisting tensioncompression residual in-plane stresses that are released in the slice. We performed this method on tissues, such as the normal kidney and lymph node micro-metastasis, and observed negligible deformation after slicing (Fig. 3E-F), consistent with the results of the planar cut method (Fig. 1C).

The release of elastic energy via slicing results in surface area expansion of the slice. The overall area expansion after slicing represents the area strain, a quantitative index for inplane solid stresses and the stored elastic energy in the slice. We defined the area expansion ratio as:

$$
I_{E R}=\frac{A_{\text {tumor }}-A_{\text {blank }}}{A_{\text {blank }}}
$$

where $A_{\text {tumour }}$, is the surface area of the tumour slice after the elastic energy is released and obtained from the $3 \mathrm{D}$ image of the slice, and $\mathrm{A}_{\text {blank }}$ is the reference area of the tumour slice before slicing, obtained from the empty space left in the agarose gel (Fig. 3H, I). The expansion ratio has been quantified as $30.7 \pm 5.8 \%$ for breast tumour, $34.0 \pm 4.9 \%$ for PDAC, and $17.2 \pm 1.2 \%$ for lymph node with breast cancer macro-metastasis, which are significantly higher than the control tissues: $7.0 \pm 1.9 \%$ for kidney, and $5.0 \pm 1.2 \%$ for lymph node with micro-metastasis (Fig. 3J and Fig. S4). It should be mentioned that in this method, the tumour undergoes consecutive cuts prior to reaching the desired slice in the tumour. In doing so, the out-of-plane stresses $\left(\sigma_{\mathrm{zz}}\right)$ are fully released similar to the cut method. In addition, the in-plane stresses $\left(\sigma_{\mathrm{xx}}\right.$ and $\left.\sigma_{\mathrm{yy}}\right)$ are also partially released as shown in the example in Fig. S3. For the representative case of MMTV-M3C model of breast cancer, we showed that the slicing method releases the majority of the in-plane stresses compared to the relaxation of in-plane stresses that occurs before the slicing. The average area strain obtained via slicing method in the MMTV-M3C model is $I_{\mathrm{ER}}=30.7 \%$, which results in $\left\langle\sigma_{\text {in-plane }}\right\rangle=0.27 \mathrm{kPa}$. (The Young's modulus $E=0.87 \mathrm{kPa}$.) However, the average $\sigma_{\mathrm{xx}}$ and $\sigma_{\mathrm{yy}}$ that are released by the cuts prior to the desired slicing are $0.069 \mathrm{kPa}$ and $0.078 \mathrm{kPa}$ (Fig. S3), respectively, no larger than one-third of $\left\langle\sigma_{\text {in-plane }}\right\rangle$, which shows that the majority of the in-plane stresses are relaxed via slice method, and are thus reported as area strain $I_{\mathrm{ER}}$ (Eq. 3).

\section{Evolution of solid stress and stiffness during tumour growth}

Investigating the accumulation of solid stress as a function of tumour growth provides invaluable information regarding both the genesis and implications of solid stress. We used the slice method to measure the expansion ratio $I_{\mathrm{ER}}$ (Eq. 3), induced by the relaxation of solid stress, in MMTV-M3C breast tumours for tumours ranging in size from 2 to $7 \mathrm{~mm}$ in 
diameter (Fig. 4A). The elastic modulus of the tumours was also measured via atomic force microscopy (AFM)-based indentation for a similar range of tumour size. The expansion ratio (Eq. 3), induced by the relaxation of in-plane stresses in the slice, increased significantly with increasing tumour diameter (Fig. 4A, B). Tumours with small diameters (2-3 mm) did not show a significant increase in area after slicing while the larger tumours showed an area expansion of up to 30-40\%. Notably, the AFM-based indentation modulus did not show any significant trend with tumour diameter (Fig. 4C). This confirms that solid stress and elastic energy can be considered as distinct mechanical abnormalities from stiffness, long known to be higher in many tumours compared to normal tissues.

The collagen I network becomes more organized and more localized at the periphery with increasing tumour size in MMTV-M3C breast tumour model, consistent with the increase in solid stress. As shown via immunofluorescence analysis, the collagen I network is uniformly and randomly distributed in smaller tumours $(d \sim 2 \mathrm{~mm}$; Fig. 4D) and becomes more localized and organized at the periphery in larger tumours $(d \sim 7 \mathrm{~mm})$. To quantitatively describe this collagen I localization effect, we defined a collagen spread radius, $R_{\mathrm{c}}$, as the radius of an area that contains $50 \%$ of the positively stained collagen I. This radius increases significantly with tumour diameter (Fig. 4D), consistent with a similar increase in area expansion ratio (Fig. 4B). The highly oriented collagen (Fig. S5) with increased density at the periphery implies that the periphery of the tumour is experiencing tensile stresses. This tensile stress in the periphery, consistent with the stress map in Fig. 1D, is hypothesized to originate from the direct contraction of carcinoma associated fibroblasts [30, 31] or to resist the compressive forces emanating from the volume increase from cell proliferation and ECM deposition.

While the solid stress increased with tumour size, the fraction of perfused vessels showed an opposite trend (Fig. 4E; see Fig. S6 for representative images), consistent with the hypothesis that solid stress can compress blood vessels and decrease tumour perfusion [9, $14,19]$. These data also suggest that stiffness, showing no significant trend with tumour growth for the range of tumour diameters in this study, is not a major contributor to compression and collapse of intratumour blood vessels.

\section{Physical role of surrounding tissue in solid stress in tumours}

Until now, we estimated the solid stress and elastic energy of tumours ex vivo, independent of the surrounding normal tissue. Here, via in situ estimation of solid stress, we show that the resistance to the growth from the surrounding tissue also significantly contributes to the solid stress in the tumour - as predicted previously by our mathematical model [32, 33]. To measure the in situ solid stresses, we perform a core biopsy in the tumour to create a cylindrical void. The changes in diameter of the cylindrical void, measured by ultrasonography, represent a one-dimensional stress profile in the tumour. We first characterized this method ex vivo to release the solid stress in the MMTV-M3C model of breast tumours: the cylindrical void reduced in diameter in the center and increased in diameter at the periphery of the tumour (Fig. 5B). This deformation profile indicates compressive stresses in the center, and tensile stresses in the periphery of the tumour, consistent with the results obtained by the planar cut method described above (Fig. 1D). As 
expected, the diameter profile of the biopsy void remained unchanged in control tissues such as kidney and liver, in which the solid stress is negligible (Fig. 5C, D).

In addition to the void left by the biopsy, the biopsy core also contains useful information about the state of solid stress in the probed tissue. The tip of the core shrinks due to the tensile contraction at the periphery, and the diameter in the middle region increases due to release of compressive stresses (Fig. 5B). The biopsy cores from the control tissues such as kidney and liver do not deform, again due to the negligible solid stress and elastic energy in these tissues (Fig. 5C, D).

Utilizing this method for in situ stress measurement, we showed that the solid stress in $e x$ vivo brain tumours (glioblastoma, U87) is lower in magnitude and different in profile than in tumours surrounded by brain and cranium (in situ; Fig. 5E, F, G). We used the same glioblastoma model (U87) for which the 2D stress map was quantified in Fig. 1, and released the solid stress by biopsy punch while the tumour is still surrounded by brain and cranium. For in situ measurements, we first made a 2-mm diameter hole in the cranial window (Fig. 5H), through which we accessed the tumour with a 1.5-mm biopsy punch. The biopsy void reduced in diameter at regions close to the tumour rim, consistent with the 2-D map of deformation (Fig. 1D), but in contrast to the primary breast tumours where the maximum deformation occurs in the center (Fig. 5B). For ex vivo measurements, the tumour was surgically resected from the brain, embedded in $2 \%$ agarose, and the stress was released by the same biopsy technique (Fig. 5G). We observed an overall smaller deformation for the ex vivo compared with the in situ tissues, and these differences in deformation were significant in regions closer to the outer rim of the tumour (Fig. 5F). Using a mathematical model, the maximum radial stress for the in situ case was estimated as $0.1 \mathrm{kPa}$ in compression, as compared to $0.02 \mathrm{kPa}$ in the ex vivo case.

\section{Discussion}

Based on the fundamental concept that tissues containing solid stress undergo deformation after release of physical confinement, we developed three different experimental techniques and appropriate mathematical models to quantify solid stress and elastic potential energy in solid tumours. The first method, based on releasing solid stress at a plane created by bisecting the tumour, reveals the 2-D spatial distribution of solid stress. This method allows us to distinguish regions with tensile stresses, mostly at the periphery, from the central regions of the tumour that experience mainly compressive stresses. The second method, based on releasing solid stress by creating thin tumour slices, provides a sensitive method to quantify low levels of solid stress in small specimens, such as small metastatic lesions in mice. The third method, based on releasing solid stress by core biopsy, is capable of simultaneous in situ and ex vivo estimations of solid stress. The in situ feature of this method allowed determination of the contribution of the surrounding tissue to the intratumoural solid stress. Importantly, we found that biopsy cores also undergo ex vivo stress-induced deformation, and could potentially be used for measurement of solid stress in the clinic (See Table S1 for capabilities and limitations of each method). These methods overcome the previous technical challenges in measuring these biomechanical abnormalities. 
Reliable and precise quantification of solid stress and elastic energy has the potential to promote the discovery of new signalling pathways and therapeutic strategies. The repurposing of losartan [14], an angiotensin receptor inhibitor, and PEGPH20 [18], a recombinant hyaluronidase, in cancer therapies is partly based on releasing the mechanical forces exerted on blood vessels in tumours by targeting collagen I and HA. The administration of both drugs resulted in increased efficiency of chemotherapy in preclinical models, leading to two phase II clinical trials [20,21]. Similar to our earlier method to estimate solid stress [9], the methods developed here could also be used to compare agents that can lower solid stress. Moreover, the interplay between obesity and the cancer immune microenvironment would also benefit from the characterization of solid stress. As we recently found in PDAC tumours [34, 35], synergies between adipocytes, immune cells and fibroblasts lead to an exacerbated desmoplastic microenvironment during obesity, further promoting blood-vessel compression. This is arguably the most important consequence of solid stress demonstrated to date.

Solid stress and elastic energy can also be used as prognostic and diagnostic markers. Factors such as tumour growth pattern and collagen architecture, both closely associated with solid stress, have been shown to have prognostic values. Growth pattern, histologically categorized as pushing vs. replacing phenotypes, has been linked to overall survival [36] and recurrence risk [37]. As shown in Fig. 2, colorectal cancer liver metastases with pushing growth pattern have higher elastic energy compared to pancreatic cancer liver metastases with replacing growth pattern (Fig. S7). Also, collagen orientation and alignment have been shown to correlate with prognosis in breast cancer patients [38]. As shown, the collagen network in our breast tumour model becomes more organized as solid stress increases with tumour growth. Quantification and association of solid stress with growth pattern and collagen architecture suggest that solid stress may reveal the mechanisms underlying these histological patterns, and lead to further prognostic biomarker discoveries.

The needle biopsy and planar cut methods both provide quantitative measurements of solid stress, and have the potential to be utilized in the clinic. Biopsy samples are obtained routinely during diagnosis and treatment of cancer; as we showed in Fig. 5A and B for the needle biopsy method, the biopsy core contains useful information about solid stress in tumours. This evaluation of biopsy core solid stress could be initially performed in clinical trials as a new biomarker, and also to investigate the mechanism underlying the link between solid stress and collagen orientation and growth pattern. The planar cut method currently requires the sample to be embedded in agarose and imaged via high-resolution ultrasound; clinically, this procedure may affect the routine processing of tumours by pathologists. To facilitate this procedure, we are working with pathologists to develop methods of generating 3-D reconstruction of stress-induced deformation of patient specimens utilizing laser scanning technologies. This methodology will eliminate the agarose embedding step, shorten the imaging time, provide the strain field in a larger field of view in the tumour and its surrounding tissue, and could be coupled with the routine pathological processes on freshly excised tumours. Using fiducial markers, the solid stress maps would be matched and compared to histological images from the same cut plane. We expect that clinically accessible and quantitative measurement of solid stress and elastic energy will provide new 
prognostic and diagnostic markers, similar to stiffness $[39,40]$ and interstitial fluid pressure [41-43], two mechanical abnormalities, which have been used as prognostic biomarkers.

Finally, we utilized the above methods to demonstrate that elevated solid stress and stiffness are two distinct biomechanical abnormalities. This important distinction implies that attempts to normalize the tumour's mechanical environment should not be limited to decreasing stiffness. Two of our independent but consistent findings demonstrate that (i) primary and metastatic tumours can have significantly different levels of solid stress despite similar stiffness values (Fig. 2E, G), and (ii) solid stress increases with tumour growth, even though stiffness did not change in our tumour models (Fig. 4B, C). These findings clearly suggest that the external compressive or tensile solid stresses that tumour cells feel may be independent of the cellular focal adhesion forces that are generated in response to the stiffness of the matrix in their vicinity. With the methods and results shown here, we now have the tools to investigate how cancer and stromal cells sense and respond to tensile or compressive forces to complement our understanding of the effects of altered stiffness [44].

\section{Methods}

\section{Planar cut method}

Releasing stress-The $2 \%$ agarose is made from low gellation temperature agarose (Sigma-Aldrich) mixed with PBS. The agarose solution is in a liquid state at a temperature of $40{ }^{\circ} \mathrm{C}$. The freshly excised tumour is first washed with PBS, then immersed in the liquid agarose inside a custom-made aluminum cast. The cast is then cooled down in ice-water. After solidification of the agarose gel, the tumour-agarose construct is removed from the cast, and is manually cut at the plane of interest with a razor blade. The solidified agarose that surrounds and holds the tumour allows the tumour to be cut with minimal displacement relative to the gel. One half of the tumour is removed from the gel and fixed in $4 \%$ formaldehyde for histological analysis, and the other half, still inside the agarose construct, is kept immersed in PBS for at least 10 minutes to allow the tissue to relax and deform due to the release of solid stress and elastic energy. The same method was used for control tissues.

Tissue imaging and post-processing-The tissue deformation map is imaged via high-resolution ultrasound, Vevo 2100 system (FUJIFILM Visual Sonics, Toronto, Canada), on the half of the tumour-agarose construct that is immersed and relaxed in PBS. We used the probe MS550S for large samples such as primary breast tumour (depth of view $=13 \mathrm{~mm}$, frequency $=32-56 \mathrm{MHz}$ ), and the probe MS700 for smaller samples such as brain tumours (depth of view $=9 \mathrm{~mm}$, frequency $=30-70 \mathrm{MHz}$ ). The high-resolution ultrasound provides a resolution of $20-30 \mu \mathrm{m}$. For higher resolution and smaller samples, optical systems such as the optical frequency domain imaging (OFDI) system [29] (resolution $=1 \mu \mathrm{m}$, depth of view $=2-3 \mathrm{~mm}$ ) can be used. The 3-D images are exported from VisualSonics software to MATLAB (The MathWorks, Natick, MA) for post-processing. The post-processing includes smoothing, which is performed with moving averaging on the top (cut plane) and bottom surfaces of the tumour to remove any noise introduced by ultrasound imaging. The top and bottom surfaces are exported to SolidWorks (Waltham, MA) for 3-D reconstruction of the 
surfaces into a solid object. This solid object is then exported to the compatible finite element model, as described below.

Mathematical Modeling-In the incision method, to translate the stress-induced deformation to solid stress, we developed a mathematical model using the general purpose commercial finite element software ABAQUS (Version 6.9, SIMULIA, Providence, RI). The 3-D geometry of the tumour is imported from the SolidWorks software as described above. We used the element type C3D10, a quadratic tetrahedral element with two elastic parameters: the Young's modulus and the Poisson's ratio, which are obtained as described below. The assumptions for the use of Eq. (1) are justified in Supplementary Note 1. We have shown that the viscoelastic time constant is on the order of 10 min (Fig. S8 and S9) for the length scales comparable to tumour diameter (this time constant is mainly determined by the Young's modulus and hydraulic permeability in poroelastic materials [45, 46]). In contrast, the biological processes that give rise to solid stress in tumours, such as proliferation, ECM deposition and cell contraction, occur on a time scale ranging from several hours to days. Since this is much longer than the viscoelastic time constant, the equilibrium mechanical properties that we are measuring reflect the appropriate properties in the generation and application of solid stress.

The agarose gel is solidified from a liquid state under no external or internal residual stress. Thus, the cut surface in the agarose gel provides the reference plane that represents the original plane of the tumour tissue before stress relaxation in the tumour. A displacement profile is assigned to the top deformed surface of the tumour to translate it back to the original reference plane. This assigned deformation is only in the direction perpendicular to the surface of the tumour (z-direction), and the tumour top surface is free to move parallel to the surface direction ( $\mathrm{x}-\mathrm{y}$ plane). This displacement assignment translates the tumour geometry from the relaxed state (deformed surface) to the original stresses state prior to the cut (flat surface). The stress component $\sigma_{\mathrm{zz}}$ that is generated after the displacement assignment is reported as the solid stress in the intact tumour. For ex vivo applications of incision method, the boundary condition on the bottom surface of the tumour is defined as zero force. This assumption is justified for cases such as mouse models of breast tumours where the solid stress field is mainly generated internally, and the tumour is not experiencing any major external confinement or resistance. For in situ application of the incision method, a contact surface is defined between the tumour and the surrounding normal tissue. In these cases, such as with brain tumours, the normal tissue is also modeled as an elastic material with known Young's modulus, obtained as described below. The solid stress component in the direction perpendicular to the cut surface (z-direction) $\sigma_{\mathrm{zz}}$, is reported. The model also reports the elastic energy density, equivalent to the work done to displace the deformation back to the original coordinates, normalized by the tumour volume.

\section{Slicing method}

Releasing solid stress-The tumour is embedded in $2 \%$ agarose gel as described in the incision method, except that the solidification occurs in a stainless steel cast provided by the commercial compresstome, VF-300 (Precisionary Instruments Inc., San Jose, California). The tumour-agarose construct, fully immersed in PBS, is then sliced via the compresstome 
with the desired thickness in the range of 100 to $500 \mu \mathrm{m}$. The tumour slice is detached from the agarose slice, and leaves an empty area in the agarose gel. This empty area, called the "blank" in the manuscript, represents the tumour area prior to stress relaxation by slicing. The detachment of the tumour slice from the surrounding agarose is done manually with a pair of sharp tweezers; in some cases, detachment occurs spontaneously. The slice is kept immersed in PBS for at least 10 minutes while the solid stress releases. As a result of stress relaxation, the sample slice undergoes expansion in area through different modes of bending and buckling.

Imaging and post-processing-The sample slice and the blank agarose gel are imaged in 3-D via high-resolution ultrasound (for larger tumours), or the optical system OFDI (for small samples such as lymph node). The surface area of both the sample slice and the blank are then calculated by custom in-house MATLAB codes. The change in the ratio of the surface area after stress relaxation to the surface area before stress relaxation (equal to the blank area) is defined as an index of solid stress and elastic energy.

\section{Needle Biopsy Method}

Releasing solid stress-Tumour solid stress is released by punching a hole with rotating biopsy punches (Integra-Miltex, Plainsboro, NJ) with diameters $1.5-3 \mathrm{~mm}$. The rotating punch is made by detaching the stainless steel blade of the biopsy punch from the plastic holder, and rotating it with a Dremel rotary tool (Dremel, Racine, WI). The rotary punch reduces cutting artifacts by minimizing the mechanical disturbance caused otherwise by non-rotating punching. In ex vivo cases, the sample is either held gently by tweezers or embedded in $2 \%$ agarose to be secured during the punch. For in situ cases, such as brain tumours, we made a 2-mm diameter hole in the plastic coverslip of the cranial window without disturbing the underlying brain tissue. Through this hole in the cranial window, we then punched the brain tumour with a $1.5 \mathrm{~mm}$ punch. The samples are left submerged in PBS at least for 10 minutes, the time needed for stress relaxation. The biopsy core, which also changes in diameter due to solid stress, is preserved similarly in PBS.

Imaging and post-processing-The stress-induced deformation and the sample geometry are imaged by high-resolution ultrasound as described in the incision method. Using custom in-house, semi-automated MATLAB codes, we calculated the area of the biopsy hole along the punch axis, from which the mean hole diameter is calculated.

Mathematical modeling-In the needle biopsy method, an axisymmetric finite element code is developed in ABAQUS to translate the stress-induced deformation to solid stress. A deformation profile is assigned that transforms the deformed punch profile to the original profile before the stress relaxation. The original hole profile is a simply determined by the biopsy punch diameter, which is uniform along the hole axis (z-axis) and equal to $1.5 \mathrm{~mm}$ for small samples such as brain tumours, and $2-3 \mathrm{~mm}$ for larger samples. In the ex vivo cases, a zero force boundary condition is prescribed at the outer surface of the tumour to simulate the physiological conditions. For in situ cases, a contact surface is defined between the tumour and the normal tissue, such is brain, with known elastic properties. We used the four-node axisymmetric element CAX4R for both the tumour and surrounding tissue. The 
materials properties of both the tumour and the surrounding tissue are obtained via micro and macro measurements of stiffness, as described below. The stress $\sigma_{\mathrm{rr}}$, the component of solid stress on the cut surface, in the direction perpendicular to the axis of the hole (zdirection), is reported. The mathematical model also reports the elastic energy density, equivalent to the work done to displace the deformation back to the original coordinates, normalized by the volume of the tumour.

\section{Micro and macro measurement of stiffness}

The stiffness of the tumours was measured via atomic force microscopy (AFM) in microscale as indentation modulus, and in macroscale as unconfined compression modulus. For both cases the measurement has been done within $1 \mathrm{hr}$ after excision of the tumours. The samples have been maintained in phosphate-buffered saline (PBS) with protease inhibitors (Complete Protease cocktail tablets, Roche Applied Science, Indianapolis, IN) at $4{ }^{\circ} \mathrm{C}$ before testing. All the measurements were performed in near-physiological PBS at ambient temperature $\left(25^{\circ} \mathrm{C}\right)$.

AFM-based measurement of indentation modulus-The indentation moduli of tumours were quantified using an Asylum MFP3D atomic force microscope (Asylum Research, Santa Barbara, CA). We used polystyrene colloidal probe tips with end radius $R \sim$ $12.5 \mu \mathrm{m}$ (Polysciences, Warrington, PA) attached to tipless cantilevers with nominal spring constant $k \sim 0.12 \mathrm{~N} / \mathrm{m}$ (Bruker, Camarillo, CA). The colloidal probes were attached to the cantilever by the lift-off process: a dot of glue (M-Bond 610, Structure Probes/SPI Supplies, West Chester, PA) was placed on a tipless cantilever by making quick contact between the cantilever and a thin layer of glue $(1 \mu \mathrm{l})$ spread over a $10 \times 10$-mm mica surface. We then made immediate contact between the tip of the cantilever and a colloid probe resting on a glass slide and waited for $1 \mathrm{~min}$ with the cantilever pushing against the colloid. This process was followed by heat curing for $2 \mathrm{~h}$ at $150^{\circ} \mathrm{C}$. For each probe tip, the exact spring constants of the cantilevers were directly measured using the thermal calibration method [47]. The relationship between the detected voltage and the applied force was calibrated by bringing the cantilever in contact with a glass slide and calculating the slope of the voltagedisplacement curve. The displacement, $d$, was translated to force, $F$, using Hooke's Law ( $F=$ $k d$ ). The indentation was performed under a force control scheme ( $\max$ force $\sim 20 \mathrm{nN}$ ), limiting the indentation depths to $0.5-3 \mu \mathrm{m}$. The tip displacement was obtained by subtracting the cantilever deflection from vertical movement of the piezo. An indentation approach velocity of $2 \mu \mathrm{m} / \mathrm{s}$ ensured probing the elastic modulus at a lower rate, close to equilibrium condition.

The effective indentation modulus $E_{\text {ind }}$ was computed using Hertzian contact mechanics models via least-squares linear regression of the experimental loading force-displacement curves. For the spherical colloidal probe tip with end radius $R$ on the sample with thickness $h \gg R$ (here, $h \sim 5 \mathrm{~mm}, R \sim 12.5 \mu \mathrm{m}$ ),

$$
F=\frac{4}{3} \frac{E_{\text {ind }}}{\left(1-v^{2}\right)} R^{\frac{1}{2}} D^{3 / 2}
$$


where $F$ is the indentation force, $D$ is the indentation depth, and $v$ is the Poisson's ratio (we assumed $v=0.1$, as measured in other soft tissues $[48,49])$. A representative forcedisplacement curve is shown in Fig. S10.

Macroscale unconfined compression test-The Young's moduli of tumours were determined by unconfined compression tests [50]. Slices of freshly excised tissue, $3 \mathrm{~mm}$ in diameter and $\sim 2$-mm thick, were placed in an unconfined compression chamber, submerged in physiological saline. The chamber was mounted in an ultrasensitive servo-controlled materials tester (Dynastat Mechanical Spectrometer; IMASS, Hingham, MA). Each specimen was compressed by 5 percent of the original height in ramps of $20 \mathrm{~s}$ and allowed to relax for $20 \mathrm{~min}$. Four successive measurements were performed on each tissue slice. The Young's modulus was determined as the ratio of the linear fit to stress-strain data (Fig. S8)

\section{Cell culture}

Cells (MMTV-M3C, AK4.4, SL4, U87, 4T1) were cultured in DMEM (ATCC 30-2002) medium containing $10 \%$ fetal bovine serum. Cells were harvested at $\sim 80 \%$ confluency, washed twice with phosphate buffered saline (PBS), counted and resuspended in DMEM prior to injection. All cell lines repeatedly tested negative for mycoplasma using the Mycoalert Plus Mycoplasma Detection Kit (Lonza, Allendale, NJ), authenticated before use by IDEXX laboratories (North Grafton, MA).

\section{Tumour models}

Primary tumour models-MMTV-M3C cells $(\mathrm{Her} 2+)$ were kindly provided by Dr. Peigen Huang (Massachusetts General Hospital, Boston), and were isolated from lung metastases of mammary adenocarcinomas in mice generating spontaneous mammary tumours (MMTV-PyVT). Orthotopic breast tumours were generated by implanting a small piece $\left(1 \mathrm{~mm}^{3}\right)$ of viable tumour tissue (from a source tumour in a separate animal) or by injection of $1 \times 10^{5}$ cells into the mammary fat pad of a 6-8 week-old female FVB (MMTVM3C). AK4.4 cells ( Kras ${ }^{G 12 D}$ and $p 53^{+/-}$) were kindly provided by Dr. Nabeel Bardeesy (Massachusetts General Hospital, Boston), and were isolated from mice generating spontaneous pancreatic tumours (Ptf1-Cre/LSL-Kras ${ }^{G 12 D} / \mathrm{p} 53$ ). Orthotopic pancreatic tumours were similarly generated by implanting a small piece $\left(1 \mathrm{~mm}^{3}\right)$ of viable tumour tissue into the pancreas of a 6-8 week-old male FVB mouse (AK4.4 model) mouse. The SL4 mouse colorectal cancer cells were a generous gift from Prof. Irimura [51]. For the orthotopic SL4 model, $5 \times 10^{5}$ cells in $10 \mu \mathrm{L}$ of PBS/Matrigel complex were injected into the cecal wall between the serosa and mucosa from the serosal side using an insulin syringe with a $27 \mathrm{G}$ needle. For the glioblastoma model, cranial windows were implanted into nude mice as previously described [52], and the mice were allowed to recover one week before tumour implantation. U87 fragments $(0.2-0.3 \mathrm{~mm}$ diameter; from a source tumour in a separate animal) were implanted into the left cerebral hemisphere $0.5-1 \mathrm{~mm}$ deep in a 6-8 week-old female nude mouse. All animal procedures were carried out following the Public Health Service Policy on Humane Care of Laboratory Animals and approved by the Institutional Animal Care and Use Committee of Massachusetts General Hospital. 
Liver metastasis model-mice were anesthetized with an intraperitoneal injection of ketamine $(100 \mathrm{mg} / \mathrm{kg})$ and xylazine $(10 \mathrm{mg} / \mathrm{kg})$. A left flank incision was made through the skin and abdominal wall layer, and the spleen was gently exteriorized. The middle of the spleen was ligated with a suture prior to transsection, with the goal of preserving the vascular pedicles to both hemispleens. $1 \times 10^{5}$ cells (AK4.4 or SL4) in $100 \mu \mathrm{L}$ DMEM were injected slowly into the distal lobe with a 30-gauge needle. After 10 minutes the hemispleen used for tumour cell injection was resected. The remaining spleen was placed back into the abdomen. The abdominal wall was closed with a continuous suture. The skin was closed with surgical staples.

Lymph node spontaneous metastasis- $2 \times 10^{5} 4 \mathrm{~T} 1$ cells in DMEM were injected into the 2 nd mammary fat pad of syngeneic BALB/c mice. On day 15 post-injection, the primary tumour was resected. Animals were sacrificed $2-3$ weeks post-resection to evaluate metastatic lymph nodes.

\section{Histology}

Prior to sacrifice, mice were slowly ( 2min) injected with $100 \mathrm{ml}$ of $1 \mathrm{mg} / \mathrm{ml}$ FITC conjugated lectin (Vector Labs), administered via the tail vein 5 min before tumour removal, long enough for FITC-lectin to reach perfused vessels. The tumours were then excised, fixed in $4 \%$ formaldehyde in PBS overnight, and embedded in paraffin blocks. Transverse tumour sections, $5 \mu \mathrm{m}$ thick, were immunostained with antibodies for endothelial marker CD31 (DIA310, Dianova, 1:100 dilution) and counterstained with DAPI (Vector Labs). Collagen I and hyaluronan were, respectively, detected using the LF-68 antibody (1:500 dilution) provided by Dr Larry Fisher (NIDCR) and a biotinylated hyaluronan proteoglycan fragment (385911, Calbiochem). For the detection of collagen I, the paraffin-embedded sections were treated with a pH-9.0 trypsin antigen retrieval solution and counterstained with DAPI.

Staining for aSMA (C6198 antibody, Sigma, 1:100 dilution) was also carried out. The entire tumour section at 10x magnification was taken from each slide using a confocal microscope (Olympus) or slide scanner (TissueFAXS). For vascular analysis, vessels were segmented using a custom, semi-automated tracing program developed in MATLAB, allowing the analysis of each vessel according to its size and staining intensity. Tumour perfusion was assessed by identifying the fraction of CD31+ vessels that were also positive for FITClectin, indicating that those individual vessels were perfused prior to sacrifice, and therefore functional and uncompressed.

\section{Collagenase treatment}

Collagenase (Sigma-Aldrich) was prepared by dissolving $10 \mathrm{mg}$ collagenase in $10 \mathrm{~mL}$ DMEM. After excision, the tumour was placed in the collagenase solution remaining at $37^{\circ} \mathrm{C}$ for $2 \mathrm{~h}$ before solid stress measurement.

\section{Statistical analysis}

The data are presented as mean \pm standard error of the mean. Groups were compared using an unpaired, two-sided Student's $t$-test (due to independent sampling) with equal or unequal variance. The variance similarity was tested via F-test with $p=0.05$. All the results are based on pilot studies (not reported) preceding the main experiments (reported). The sample size 
for the main experiments were determined from the pilot studies. Mice were size matched as appropriate for each experiment; no additional blinding nor randomization was required for the animal studies.

\section{Supplementary Material}

Refer to Web version on PubMed Central for supplementary material.

\section{Acknowledgments}

We thank Sylvie Roberge, Carolyn Smith, Julia Kahn and Mark Duquette for technical assistance. We also thank Dr. Peigen Huang, Dr. Nabeel Bardeesy, and Prof. Irimura for providing MMTV-M3C, AK4.4 and SL4 cells, respectively. This work was supported in part from funding from the National Cancer Institute (P01-CA080124), NCI Outstanding Investigator Award (R35-CA197743) and DoD Breast Cancer Research Innovator award (W81XWH-10-1-0016) to RKJ, DP2 OD008780 to TPP and R01-HL128168 grant to LLM, TPP and JWB.

\section{Appendix}

\section{Additional information}

Supplementary information is available in the online version of the paper

\section{Author Contributions}

H.T.N. and R.K.J. designed the study; H.T.N., H.L., G.S., M.D., D.J., N.R., J.I., K.J. performed research; H.T.N., H.L., G.S., M.D., D.J., N.R., J.I., V.P.C., K.J., J.D.M., V.A., T.P.P., D.F., Y.B., F.J.H., A.J.G., J.W.B., L.L.M. and R.K.J. analyzed data; H.T.N., M.D., G.S., V.P.C., L.L.M. and R.K.J. wrote the manuscript.

\section{Data availability}

Custom scripts for MATLAB (The MathWorks, Natick, MA) are available as Supplementary Information.

\section{Competing Financial Interests}

R.K.J. received consultant fees from Ophthotech, SPARC, SynDevRx and XTuit. RKJ owns equity in Enlight, Ophthotech, SynDevRx, and XTuit and serves on the Board of Directors of XTuit and the Boards of Trustees of Tekla Healthcare Investors, Tekla Life Sciences Investors, Tekla Healthcare Opportunities Fund and Tekla World Healthcare Fund. No reagents or funding from these companies was used in these studies.

\section{References}

1. Jain RK, Martin JD, Stylianopoulos T. The role of mechanical forces in tumour growth and therapy. Annual Review of Biomedical Engineering. 2014; 16:321.

2. Chaudhuri O, Koshy ST, da Cunha CB, Shin JW, Verbeke CS, Allison KH, Mooney DJ. Extracellular matrix stiffness and composition jointly regulate the induction of malignant phenotypes in mammary epithelium. Nature Materials. 2014; 13(10):970-978. [PubMed: 24930031] 
3. Mouw JK, Yui Y, Damiano L, Bainer RO, Lakins JN, Acerbi I, Ou G, Wijekoon AC, Levental KR, Gilbert PM. Tissue mechanics modulate microRNA-dependent PTEN expression to regulate malignant progression. Nature Medicine. 2014; 20(4):360.

4. Samuel MS, Lopez JI, McGhee EJ, Croft DR, Strachan D, Timpson P, Munro J, Schröder E, Zhou J, Brunton VG. Actomyosin-mediated cellular tension drives increased tissue stiffness and $\beta$-catenin activation to induce epidermal hyperplasia and tumour growth. Cancer Cell. 2011; 19(6):776-791. [PubMed: 21665151]

5. Wirtz D, Konstantopoulos K, Searson PC. The physics of cancer: the role of physical interactions and mechanical forces in metastasis. Nature Reviews Cancer. 2011; 11(7):512-522. [PubMed: 21701513]

6. Goetz JG, Minguet S, Navarro-Lérida I, Lazcano JJ, Samaniego R, Calvo E, Tello M, Osteso-Ibáñez T, Pellinen T, Echarri A. Biomechanical remodeling of the microenvironment by stromal caveolin-1 favors tumour invasion and metastasis. Cell. 2011; 146(1):148-163. [PubMed: 21729786]

7. Tung JC, Barnes JM, Desai SR, Sistrunk C, Conklin MW, Schedin P, Eliceiri KW, Keely PJ, Seewaldt VL, Weaver VM. Tumour mechanics and metabolic dysfunction. Free Radical Biology and Medicine. 2015; 79:269-280. [PubMed: 25532934]

8. Helmlinger G, Netti PA, Lichtenbeld HC, Melder RJ, Jain RK. Solid stress inhibits the growth of multicellular tumour spheroids. Nature Biotechnology. 1997; 15(8):778-783.

9. Stylianopoulos T, Martin JD, Chauhan VP, Jain SR, Diop-Frimpong B, Bardeesy N, Smith BL, Ferrone CR, Hornicek FJ, Boucher Y. Causes, consequences, and remedies for growth-induced solid stress in murine and human tumours. Proceedings of the National Academy of Sciences. 2012; 109(38):15101-15108.

10. Boucher Y, Jain RK. Microvascular pressure is the principal driving force for interstitial hypertension in solid tumours: implications for vascular collapse. Cancer Research. 1992; 52(18): 5110-5114. [PubMed: 1516068]

11. Griffon-Etienne G, Boucher Y, Brekken C, Suit HD, Jain RK. Taxane-induced apoptosis decompresses blood vessels and lowers interstitial fluid pressure in solid tumours clinical implications. Cancer Research. 1999; 59(15):3776-3782. [PubMed: 10446995]

12. Padera TP, Kadambi A, di Tomaso E, Carreira CM, Brown EB, Boucher Y, Choi NC, Mathisen D, Wain J, Mark EJ. Lymphatic metastasis in the absence of functional intratumour lymphatics. Science. 2002; 296(5574):1883-1886. [PubMed: 11976409]

13. Padera TP, Stoll BR, Tooredman JB, Capen D, di Tomaso E, Jain RK. Pathology: cancer cells compress intratumour vessels. Nature. 2004; 427(6976):695-695. [PubMed: 14973470]

14. Chauhan VP, Martin JD, Liu H, Lacorre DA, Jain SR, Kozin SV, Stylianopoulos T, Mousa AS, Han X, Adstamongkonkul P, Popovich Z, Huang P, Bawendi M, Boucher Y, Jain RK. Angiotensin inhibition enhances drug delivery and potentiates chemotherapy by decompressing tumour blood vessels. Nature Communications. 2013:4.

15. Jain RK. Antiangiogenesis strategies revisited: from starving tumours to alleviating hypoxia. Cancer Cell. 2014; 26(5):605-622. [PubMed: 25517747]

16. Fernández-Sánchez ME, Barbier S, Whitehead J, Béalle G, Michel A, Latorre-Ossa H, Rey C, Fouassier L, Claperon A, Brullé L. Mechanical induction of the tumourigenic [bgr]-catenin pathway by tumour growth pressure. Nature. 2015; 523(7558):92-95. [PubMed: 25970250]

17. Tse JM, Cheng G, Tyrrell JA, Wilcox-Adelman SA, Boucher Y, Jain RK, Munn LL. Mechanical compression drives cancer cells toward invasive phenotype. Proceedings of the National Academy of Sciences. 2012; 109(3):911-916.

18. Provenzano PP, Cuevas C, Chang AE, Goel VK, Von Hoff DD, Hingorani SR. Enzymatic targeting of the stroma ablates physical barriers to treatment of pancreatic ductal adenocarcinoma. Cancer Cell. 2012; 21(3):418-429. [PubMed: 22439937]

19. Chauhan VP, Boucher Y, Ferrone CR, Roberge S, Martin JD, Stylianopoulos T, Bardeesy N, DePinho RA, Padera TP, Munn LL, Jain RK. Compression of Pancreatic Tumour Blood Vessels by Hyaluronan Is Caused by Solid Stress and Not Interstitial Fluid Pressure. Cancer Cell. 2014; 26(1): 14-15. [PubMed: 25026209]

20. ClinicalTrials.gov. Proton w/FOLFIRINOX-Losartan for Pancreatic Cancer. Available from: https://clinicaltrials.gov/ct2/show/NCT01821729 
21. ClinicalTrials.gov. PEGPH20 Plus Nab-Paclitaxel Plus Gemcitabine Compared With NabPaclitaxel Plus Gemcitabine in Subjects With Stage IV Untreated Pancreatic Cancer (HALO-109-202). Available from: https://clinicaltrials.gov/ct2/show/NCT01839487

22. Chuong, C., Fung, Y. Frontiers in Biomechanics. Springer; 1986. Residual stress in arteries; p. 117-129.

23. Taber LA, Humphrey JD. Stress-modulated growth, residual stress, and vascular heterogeneity. Journal of Biomechanical Engineering. 2001; 123(6):528-535. [PubMed: 11783722]

24. Campas O, Mammoto T, Hasso S, Sperling RA, O’Connell D, Bischof AG, Maas R, Weitz DA, Mahadevan L, Ingber DE. Quantifying cell-generated mechanical forces within living embryonic tissues. Nature Methods. 2014; 11(2):183-189. [PubMed: 24317254]

25. Grashoff C, Hoffman BD, Brenner MD, Zhou R, Parsons M, Yang MT, McLean MA, Sligar SG, Chen CS, Ha T. Measuring mechanical tension across vinculin reveals regulation of focal adhesion dynamics. Nature. 2010; 466(7303):263-266. [PubMed: 20613844]

26. Timoshenko, S., Goodier, J. Theory of elasticity. Vol. 412. New York: 1951. p. 108

27. Plodinec M, Loparic M, Monnier CA, Obermann EC, Zanetti-Dallenbach R, Oertle P, Hyotyla JT, Aebi U, Bentires-Alj M, Lim RY. The nanomechanical signature of breast cancer. Nature Nanotechnology. 2012; 7(11):757-765.

28. Lopez J, Kang I, You W, McDonald D, Weaver V. In situ force mapping of mammary gland transformation. Integr Biol (Camb). 2011; 3(9):910-21. Epub 2011/08/16. DOI: 10.1039/ clib00043h [PubMed: 21842067]

29. Vakoc BJ, Lanning RM, Tyrrell JA, Padera TP, Bartlett LA, Stylianopoulos T, Munn LL, Tearney GJ, Fukumura D, Jain RK, Bouma B. Three-dimensional microscopy of the tumour microenvironment in vivo using optical frequency domain imaging. Nature Medicine. 2009; 15(10):1219-1223.

30. Simon D, Horgan C, Humphrey J. Mechanical restrictions on biological responses by adherent cells within collagen gels. Journal of the Mechanical Behavior of Biomedical Materials. 2012; 14:216-226. [PubMed: 23022259]

31. Orimo A, Gupta PB, Sgroi DC, Arenzana-Seisdedos F, Delaunay T, Naeem R, Carey VJ, Richardson AL, Weinberg RA. Stromal fibroblasts present in invasive human breast carcinomas promote tumour growth and angiogenesis through elevated SDF-1/CXCL12 secretion. Cell. 2005; 121(3):335-348. [PubMed: 15882617]

32. Stylianopoulos T, Martin JD, Snuderl M, Mpekris F, Jain SR, Jain RK. Coevolution of solid stress and interstitial fluid pressure in tumours during progression: implications for vascular collapse. Cancer research. 2013; 73(13):3833-3841. [PubMed: 23633490]

33. Voutouri C, Mpekris F, Papageorgis P, Odysseos AD, Stylianopoulos T. Role of constitutive behavior and tumour-host mechanical interactions in the state of stress and growth of solid tumours. PloS one. 2014; 9(8):e104717. [PubMed: 25111061]

34. Fukumura D, Incio J, Shankaraiah R, Jain RK. Obesity and cancer: an angiogenic and inflammatory link. Microcirculation. 2016

35. Incio J, Liu H, Suboj P, Chin SM, Chen IX, Pinter M, Ng MR, Nia HT, Grahovac J, Kao S, Babykutty S, Huang Y, Rahbari N, Han X, Chauhan VP, Martin JD, Kahn J, Huang P, Desphande V, Michaelson J, Michelakos TP, Ferrone CR, Soraes R, Boucher Y, Fukumara D, Jain RK. Obesity-induced inflammation and desmoplasia promote pancreatic cancer progression and resistance to chemotherapy. Cancer Discovery. 2016:CD-15-1177.

36. Van den Eynden GG, Bird NC, Majeed AW, Van Laere S, Dirix LY, Vermeulen PB. The histological growth pattern of colorectal cancer liver metastases has prognostic value. Clinical \& Experimental Metastasis. 2012; 29(6):541-549. [PubMed: 22476470]

37. Eefsen R, Vermeulen P, Christensen I, Laerum O, Mogensen MB, Rolff H, Van den Eynden G, Høyer-Hansen G, Osterlind K, Vainer B. Growth pattern of colorectal liver metastasis as a marker of recurrence risk. Clinical \& Experimental Metastasis. 2015; 32(4):369-381. [PubMed: 25822899]

38. Conklin MW, Eickhoff JC, Riching KM, Pehlke CA, Eliceiri KW, Provenzano PP, Friedl A, Keely $\mathrm{PJ}$. Aligned collagen is a prognostic signature for survival in human breast carcinoma. The American Journal of Pathology. 2011; 178(3):1221-1232. [PubMed: 21356373] 
39. Good DW, Stewart GD, Hammer S, Scanlan P, Shu W, Phipps S, Reuben R, McNeill AS. Elasticity as a biomarker for prostate cancer: a systematic review. BJU international. 2014; 113(4):523-534. [PubMed: 23905869]

40. Choi WJ, Kim HH, Cha JH, Shin HJ, Kim H, Chae EY, Hong MJ. Predicting prognostic factors of breast cancer using shear wave elastography. Ultrasound in medicine \& biology. 2014; 40(2):269274. [PubMed: 24268451]

41. Milosevic MF, Pintilie M, Hedley DW, Bristow RG, Wouters BG, Oza AM, Laframboise S, Hill RP, Fyles AW. High tumour interstitial fluid pressure identifies cervical cancer patients with improved survival from radiotherapy plus cisplatin versus radiotherapy alone. International Journal of Cancer. 2014; 135(7):1692-1699. [PubMed: 23904154]

42. Roh H, Boucher Y, Kalnicki S, Buchsbaum R, Bloomer W, Jain R. Interstitial hypertension in carcinoma of uterine cervix in patients: possible correlation with tumour oxygenation and radiation response. Cancer Research. 1991; 51(24):6695-6698. [PubMed: 1742744]

43. Fyles A, Milosevic M, Pintilie M, Syed A, Levin W, Manchul L, Hill RP. Long-term performance of interstial fluid pressure and hypoxia as prognostic factors in cervix cancer. Radiotherapy and oncology. 2006; 80(2):132-137. [PubMed: 16920212]

44. Discher DE, Janmey P, Wang YL. Tissue cells feel and respond to the stiffness of their substrate. Science. 2005; 310(5751):1139-43. [PubMed: 16293750]

45. Nia HT, Han L, Li Y, Ortiz C, Grodzinsky A. Poroelasticity of Cartilage at the Nanoscale. Biophysical Journal. 2011; 101(9):2304-2313. [PubMed: 22067171]

46. Grodzinsky, AJ. Fields, Forces, and Flows in Biological Systems. Vol. Chapter 4. New York: Garland Science; 2011. p. 139-173.

47. Hutter JL, Bechhoefer J. Calibration of atomic-force microscope tips. Review of Scientific Instruments. 1993; 64(7):1868-1873.

48. Kiviranta P, Rieppo J, Korhonen RK, Julkunen P, Töyräs J, Jurvelin JS. Collagen network primarily controls Poisson's ratio of bovine articular cartilage in compression. Journal of Orthopaedic Research. 2006; 24(4):690-699. [PubMed: 16514661]

49. Buschmann MD, Kim YJ, Wong M, Frank E, Hunziker EB, Grodzinsky AJ. Stimulation of Aggrecan Synthesis in Cartilage Explants by Cyclic Loading Is Localized to Regions of High Interstitial Fluid Flow1. Archives of biochemistry and Biophysics. 1999; 366(1):1-7. [PubMed: 10334856]

50. Netti PA, Berk DA, Swartz MA, Grodzinsky AJ, Jain RK. Role of extracellular matrix assembly in interstitial transport in solid tumours. Cancer research. 2000; 60(9):2497-2503. [PubMed: 10811131]

51. Morimoto-Tomita M, Ohashi Y, Matsubara A, Tsuiji M, Irimura T. Mouse colon carcinoma cells established for high incidence of experimental hepatic metastasis exhibit accelerated and anchorage-independent growth. Clin Exp Metastasis. 2005; 22(6):513-21. [PubMed: 16320114]

52. Yuan F, Salehi HA, Boucher Y, Vasthare US, Tuma RF, Jain RK. Vascular permeability and microcirculation of gliomas and mammary carcinomas transplanted in rat and mouse cranial windows. Cancer Research. 1994; 54(17):4564-4568. [PubMed: 8062241] 


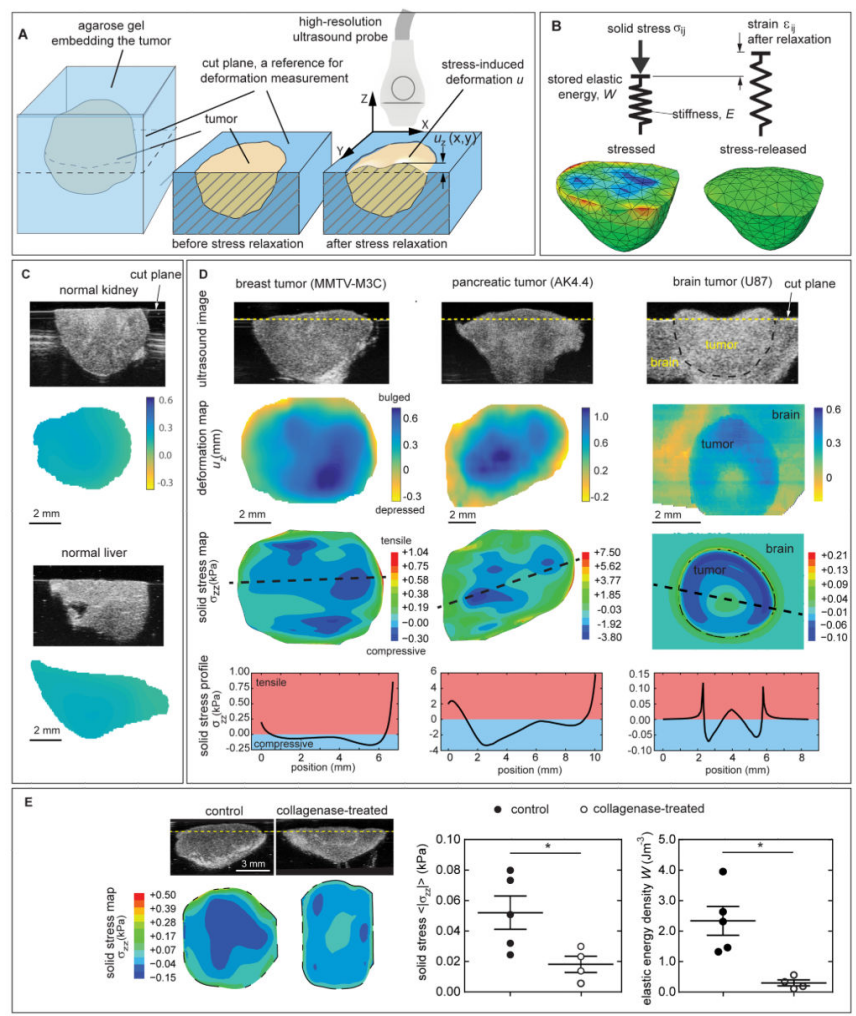

Figure 1. 2-D map of solid stress in tumours

(A) The fresh tumour is embedded in $2 \%$ agarose, liquid in $40^{\circ} \mathrm{C}$, and gellated in ice-water. The tumour-agarose construct is incised at the plane of interest to release solid stress. The release of solid stress results in the deformation field $\delta_{\mathrm{z}}(\mathrm{x}, \mathrm{y})$ with respect to the agarose reference plane; $\delta_{\mathrm{z}}$ and tumour geometry are quantified by high-resolution ultrasound (resolution of $20 \mu \mathrm{m}$ ). (B) Using a finite element model, $\sigma_{\mathrm{zz}}$, the mechanical stress in the $\mathrm{z}$ direction, and $W$, the elastic energy stored by the solid stress, are estimated from the deformation $\delta_{\mathrm{z}}$ and the Young's modulus of the tissue based on the Hooke's Law. (C) The stress-induced deformation $\delta_{\mathrm{z}}$ is uniform and negligible in stress-free normal tissues (e.g., kidney at the top and liver at the bottom). (D) A representative ultrasound image showing the stress-induced deformation and agarose reference line (yellow dashed line), the 2-D deformation field $\delta_{\mathrm{z}}$, the 2-D stress field $\sigma_{\mathrm{zz}}$, and a representative stress profile across the tumour diameter are shown for mouse models of breast tumour (MMTV-M3C), pancreatic ductal adenocarcinoma tumour (AK4.4) and brain tumour (glioblastoma U87). (E) Enzymatic depletion of collagen in (MMTV-M3C) breast tumour results in significant reduction of solid stress and elastic energy density (control: $\mathrm{n}=5$ mice; collagenase treated: $\mathrm{n}=4$ mice; mean $\pm \mathrm{SEM} ; *: p<0.05)$. The representative ultrasound image and solid stress maps are shown for control and collagenase treated tumours. 

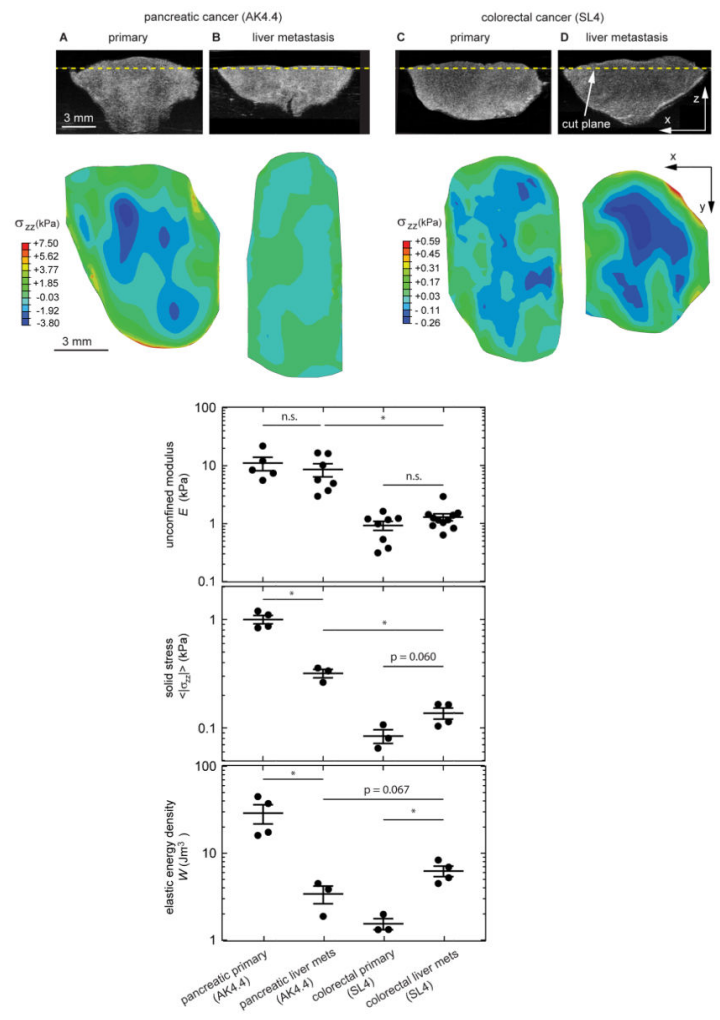

Figure 2. Solid stress and elastic energy in primary $v$ s. metastatic tumours

(A) Solid stress is mapped in size-matched primary pancreatic ductal adenocarcinoma (PDAC, AK4.4 cells; $n=4$ mice), (B) liver metastasis of PDAC (AK4.4; $n=3$ mice) (C) primary colorectal carcinoma (SL4 cells; $n=3$ mice), and (D) liver metastasis of colorectal carcinoma (SL4; $\mathrm{n}=4$ mice). Comparing (E) the Young's modulus (unconfined compression), (F) average solid stress in the z-direction, $\sigma_{\mathrm{zz}}$, and $(\mathbf{G})$ elastic energy density in these four tumour models shows that cancer cells are not the only determinant of biomechanical abnormalities: the organ and microenvironment in which the cancer cells reside are equally important in generation of solid stress and elastic energy in tumours. The data shown are mean $\pm \mathrm{SEM} ; *$ : $p<0.05$. 


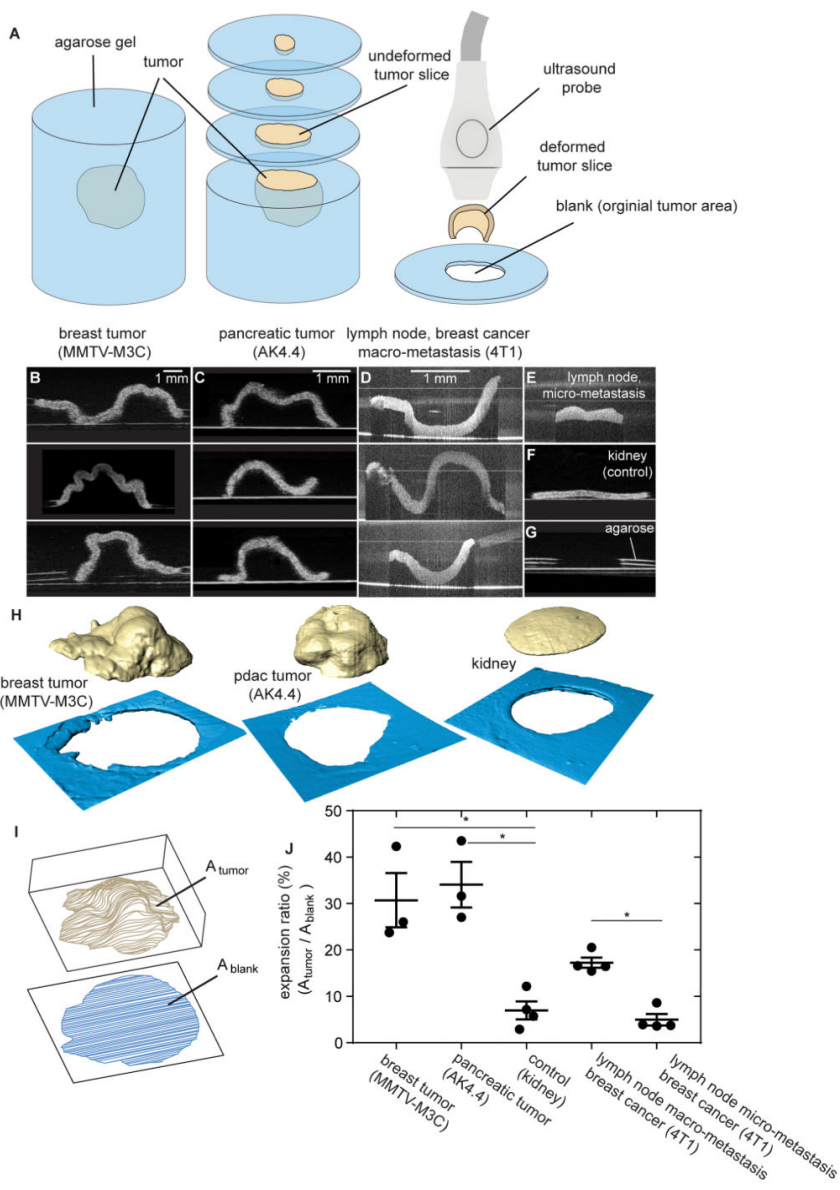

Figure 3. Creating tumour slices provides a sensitive measure of solid stress applicable to a wide range of specimen sizes

(A) The fresh tumour is embedded in liquid $2 \%$ agarose, then sliced with a compresstome and left in PBS at room temperature, so the stress-induced deformation occurs in the tumour slice. The deformed slice and the surrounding agarose disk are imaged via high-resolution ultrasound (resolution, 30 $\mu \mathrm{m}$ ) or optical frequency domain imaging (OFDI; resolution, $1 \mu \mathrm{m}$ ) for small samples (e.g., lymph node). The buckling and deformation of representative slices from (B) breast tumour (MMTV-M3C), (C) pancreatic tumour (AK4.4) and (D) lymph node with macro-metastasis of breast cancer (4T1) are shown. Tissues with negligible or low levels of stress, such as (E) lymph node with micro-metastasis and (F) kidney are shown. (G) The blank space in the agarose disk represents the area of the tumour slice before the stress relaxation. (H) The 3-D reconstruction of the slices in breast and pancreatic tumours, compared to kidney. (I) The expansion ratio, an index of solid stress and elastic energy, is defined as the ratio of the surface area of the slice after stress relaxation $A_{\text {tumour }}$ to the area of the blank in the agarose disk, $A_{\text {blank }}$ (equivalent to the area of the slice before stress relaxation). (J) The expansion ratio in breast ( $\mathrm{n}=3$ slices) and pancreatic tumours $(\mathrm{n}=3$ slices) are significantly higher than the expansion in the kidney slice ( $\mathrm{n}=3$ slices). The expansion ratio of lymph node macro-metastasis ( $n=4$ slices) is significantly higher than the one in micro-metastatic lymph node $(n=4$ slices $)($ mean \pm SEM). 


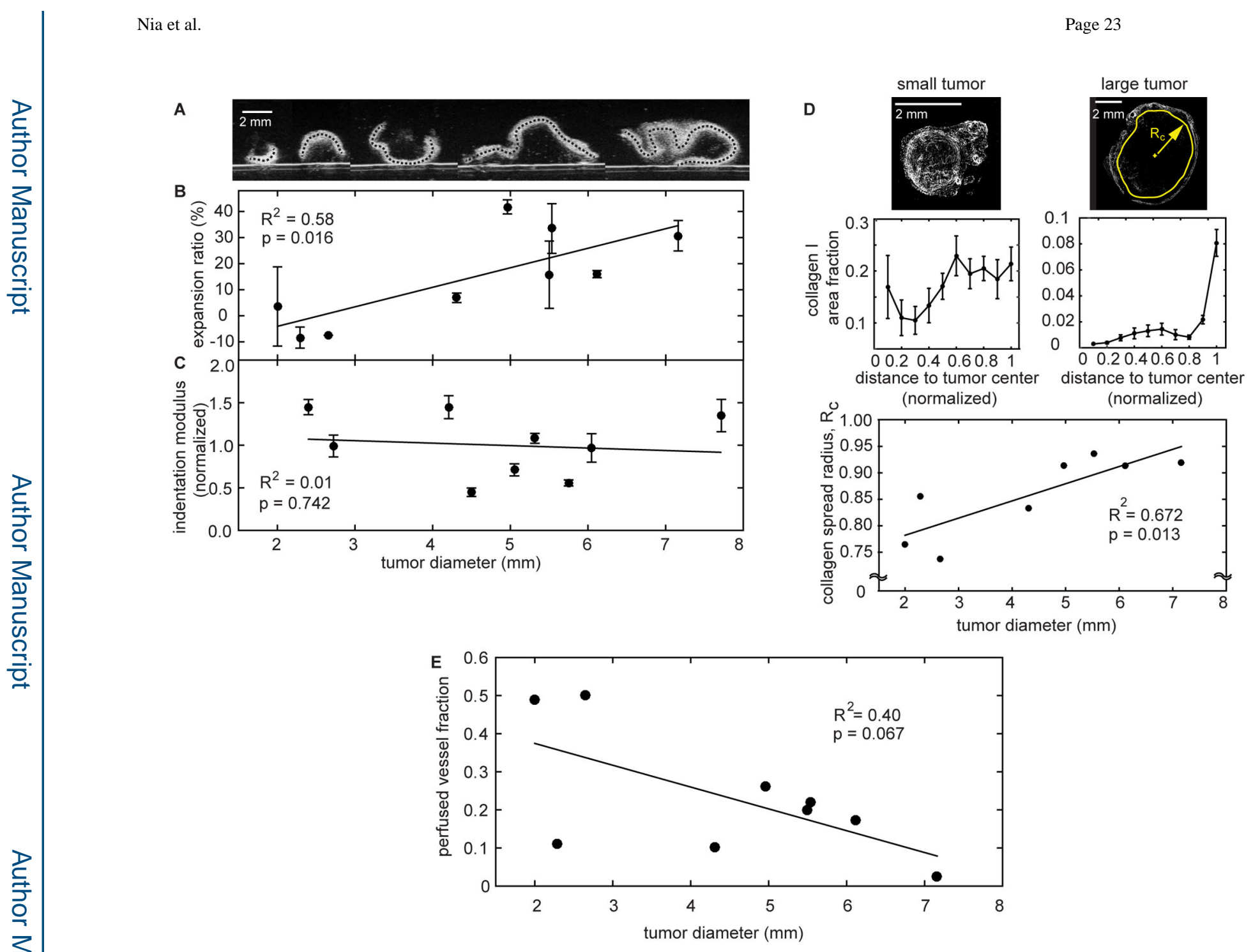

Figure 4. Evolution of solid stress and stiffness as a function of tumour size

(A) Solid stress was estimated with the slicing method in breast tumours (MMTV-M3C) with mean diameters ranging from 2-7 mm. (B) The expansion ratio - an index of solid stress - increases significantly with increasing tumour size. (C) The AFM-based indentation modulus of the same tumour model does not vary significantly with tumour size (D) Representative images of collagen I staining (white) of the MMTV-M3C tumours. The collagen content (assessed by positively stained collagen I area fraction) becomes localized to the tumour periphery with increasing tumour size. The radius $\mathrm{R}_{\mathrm{c}}$, defined as the effective normalized radius of an area that contains $50 \%$ of the positively stained collagen, increases with tumour diameter (E) The fraction of perfused blood vessels shows a decreasing trend with increasing tumour size, consistent with the hypothesis that high solid stress (which increases with tumour size, as shown in panel B) compresses blood vessels [14]. The data shown in $\mathbf{B}, \mathbf{C}, \mathbf{D}$ are mean \pm SEM. Each data point in $B$ represents $n=3$ slices from each mouse; each tumour diameter associates to a different mouse. In $\mathrm{C}$, each data point represents $n=10$ indentation sites from each mouse. In $D, n=10$ radial sectors have been quantified for each data point. 

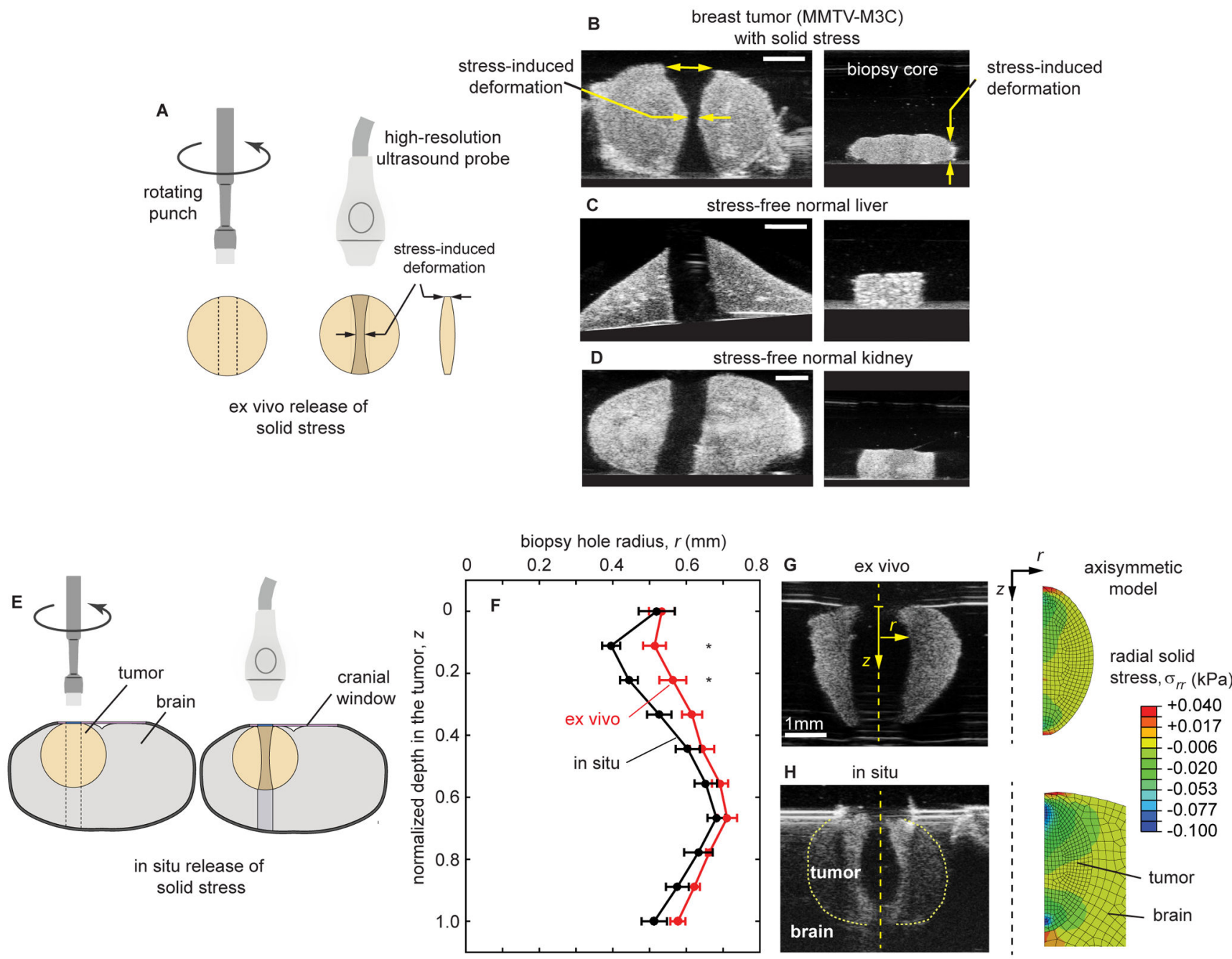

Figure 5. In situ measurement of solid stress - the normal tissue that surrounds the tumour significantly contributes to the state of stress inside the tumour

(A) The stress is released by punching a cylindrical void in the tumour. The stress relaxation results in deformation along the axis of the void: compressive and tensile stresses contract and enlarge the hole, respectively. The changes in the geometry of the biopsy core are also an indicator of solid stress. (B) This method is validated on breast tumour (MMTV-M3C). In liver $(\mathbf{C})$ and kidney $(\mathbf{D})$ - control tissues with negligible stored stress - the profile of the biopsy void and core remain constant. (E) The punching method is capable of in situ estimation of solid stress in tissues. The punch can release the solid stress in the axis of interest, without disturbing the boundary between tumour and surrounding tissue, as schematically shown for the case of brain tumour with intact normal brain and cranium. (F) The stress-induced deformation has been measured for brain tumour (glioblastoma, U87) in situ (by punching the tumour surrounded by brain, through a cranial window; $\mathrm{n}=5$ mice) and ex vivo (by punching surgically excised tumour; $n=4$ mice). $(\mathbf{G}, \mathbf{H})$ The solid stress is estimated by an axisymmetric mathematical model that uses the stress-induced deformation and the elastic properties of the tumour and brain as inputs. The distribution of $\sigma_{\mathrm{rr}}$, the stress 
at the surface of the punched hole in the $r$ direction, is shown at the original state before the deformation occurs. 